\title{
REPRESENTATION, SIMULATION AND CONTROL OF MANUFACTURING PROCESS WITH DIFFERENT FORMS OF UNCERTAINTIES
}

\author{
Hyunsoo Lee \\ Hongsuk Park \\ Amarnath Banerjee \\ Dept. of Industrial and Systems Engineering \\ 3131 TAMUS \\ Texas A\&M University \\ College Station, TX 77843, U.S.A.
}

\begin{abstract}
This paper suggests a new methodology for effectively describing and analyzing manufacturing processes with uncertainties. Uncertain information in the form of variance and vagueness are captured using probability distribution and fuzzy logic. The captured uncertainties are incorporated into a new Petri Net model referred to as Fuzzy colored Petri Net with stochastic time delay (FCPN-std). Through FCPN-std, general manufacturing uncertainties such as unclear operation rules, unfixed resource plan and processing time variances can be incorporated. This paper focuses on how FCPN-std model is generated and simulated for analyzing system performances. The procedure is illustrated using an example process. The main advantages of FCPN-std model are in the ability to capture and analyze manufacturing uncertainties, and provide an opportunity to improve process performance in the presence of uncertainties. These characteristics help modelers design exactly manufacturing process without approximations/ignorance and guide how manufacturing process can be improved through incorporated information.
\end{abstract}

\section{INTRODUCTION}

One of the main difficulties that is encountered during the modeling of manufacturing process is in how unclear manufacturing information and operation rules can be captured into the model. In its simplest form, designers often attempt to quantify uncertain information using approximation or discrete values, or ignore them completely. However, these attempts result in distorted modeling. Distorted modeling is being used to indicate inexact modeling of targeted processes through approximation or ignorance. It often results in incorrect analysis and produces unsuitable decisions. There is a need to capture and incorporate uncertain information in a manufacturing model to minimize the effect of distorted modeling, Even, manufacturing processes with clear well-defined information and rules may have uncertainties due to the random occurrence of various events, such as resource failure, change in order/demand, change of scheduling, or other emergency situations. The other reason for incorporating manufacturing uncertainties is to capture the operation knowledge and knowhow possessed by engineers and operators. The incorporation of such knowledge in a model is an essential element for reproducing and simulating successful operations of a manufacturing process. Usually, such knowledge is described using if-then clauses and these clauses may contain unclear linguistic expressions such as "very", "high", "low", "approximately" and so on. These situations make it difficult for designers to capture such information. Many researchers and designers have tried to capture these uncertainties using fuzzy logic, probabilistic information, interval theory or data-driven methodologies. Even though these methodologies have been successful in capturing some manufacturing uncertainties and were incorporated into modeling methodologies such as Petri Nets, they only covered some partial uncertainties and failed to capture many general types of uncertainties that are present in manufacturing systems. In addition, knowledge capturing techniques and modeling methodologies were not integrated well with good modeling frameworks. Table 1 shows a few examples of manufacturing uncertainties and existing knowledge capturing methodologies.

This paper uses a fuzzy colored Petri Net with stochastic time delay (FCPN-std) methodology for incorporating manufacturing uncertainties that are shown in table 1. The FCPN-std model captures manufacturing uncertainties using fuzzy logic and probabilistic functions (Lee and Banerjee 2009). The uncertain operation rules and knowledge such as resource plan or knowhow are captured using fuzzy logic, and unclear time information such as arrival time or processing times are described using stochastic time delay. These capturing methodologies are incorporated within Petri Nets. Although there are various existing fuzzy based Petri Nets and stochastic/queuing based Petri Nets, FCPN-std has the unique ability for representing general uncertainties that exist in manufacturing systems. 


\section{Lee, Park and Banerjee}

This paper focuses on how existing manufacturing process can be accurately modeled, analyzed and simulated using FCPN.A new defuzzification method is suggested for analysis and simulation of FCPN-std models. Section 2 provides a description of background and review of manufacturing uncertainties, modeling methodologies, and the definition of FCPN-std. Section 3 introduces a manufacturing process example with two parallel batch processors. Section 4 and 5 show the representation and simulation of his example using FCPN-std respectively.

Table 1: Examples of Manufacturing Uncertainties and Capturing methodologies.

\begin{tabular}{|c|c|c|}
\hline $\begin{array}{c}\text { Type of } \\
\text { Uncertainties }\end{array}$ & Examples & $\begin{array}{c}\text { Capturing } \\
\text { Methodologies }\end{array}$ \\
\hline $\begin{array}{c}\text { Unclear } \\
\text { resource plan }\end{array}$ & If process A is busy, add $\underline{1 \sim 2 \text { more resources. }}$. & $\begin{array}{l}\text { Fuzzy Logic } \\
\text { Interval theory }\end{array}$ \\
\hline $\begin{array}{l}\text { Uncertain } \\
\text { time information }\end{array}$ & $\begin{array}{l}\text { The processing time may take anywhere between } \\
\text { 30 45 minutes. }\end{array}$ & $\begin{array}{c}\text { Probabilistic functions } \\
\text { Data driven methodologies } \\
\text { Interval theory }\end{array}$ \\
\hline Operation rules & $\begin{array}{l}\text { If the bottleneck at process B is high, next arrivals } \\
\text { are handled in process B'. }\end{array}$ & Fuzzy Logic \\
\hline $\begin{array}{l}\text { Knowledge } \\
\text { /Knowhow }\end{array}$ & $\begin{array}{l}\text { If pressure is very high and temperature is } \underline{h i g h} \text {, set } \\
\text { the dial to position C. }\end{array}$ & Fuzzy Logic \\
\hline
\end{tabular}

\section{BACKGROUND AND LITERATURE REVIEW}

Manufacturing uncertainties as described in the previous section, can be broadly classified into two types of uncertainties: information with variance and information with vagueness. Information with variance can be normally captured using probabilistic distributions. Many researchers including Buzachott and Shanthikumar (1993) and Hopp and Spearman (1993) have used probability distributions to express manufacturing processes with variance, and have explained how it can be analyzed. As another type of uncertainty, information with vagueness can be depicted using fuzzy logic (Yen and Langari 1999). This concept was originally suggested by Zadeh in 1965 and since then it has been one of the popular methodologies for describing vagueness. Vagueness including linguistic variables are captured using fuzzy membership functions and they are analyzed through fuzzy operators, inference rules and various defuzzification methods.

Another important issue that exists in designing manufacturing process is in modeling methodologies itself. Even though manufacturing processes can be captured well, insufficient representation in modeling methodologies may result in the loss of captured information. There are many methodologies and software that can be used to model manufacturing processes, such as IDEF-3, Petri Nets, Message based Part State Graph (MPSG), Arena, ExtendSim, and so on. Among them, Petri Nets (PN) has been widely used as a representation, control and monitoring tool. Petri Net is considered as an useful modeling tool, since it can describe asynchronous processes and used to analyze processes by utilizing properties such as boundedness, liveness, and so on (Zhou and Venkatesh 1999).

There have been some attempts to combine Petri Net concept and methodologies for describing uncertainties. Generalized stochastic Petri Net (GSPN) and queuing Petri Net (QPN) incorporated stochastic approaches into Petri Nets (Zhou and Venkatesh 1999; Bause and Kritzinger 2002). These PNs can capture information with variance. However, they failed to capture information with vagueness. A few different approaches such as expert enhanced colored fuzzy Petri Net (EECFPN), fuzzy colored Petri Net (FCPN) and fuzzy timing high level network (FTHN) were suggested for incorporating fuzzy logic into Petri Nets (Kumar, Kumar and Tiwari 2005; Yeung, Liu, Shiu and Fung 1996; Cardoso and Camargo 1999). These PNs incorporate fuzzy logic into places and/or transitions for describing information with vagueness. Since these methodologies can capture only information with variance or information with vagueness, extended general PN (Extended GPN) was suggested for incorporating both types of information. Even though it captures uncertainties using probabilistic distribution and fuzzy logic, it does not consider multi color tokens which are needed for describing manufacturing process with multiple products or routes.

To overcome these limitations and capture general uncertainties, Lee and Banerjee (2009) have proposed FCPN-std method. FCPN-std has a 15-tuple definition: FCPN-std $=\left(\sum, \Lambda, \mathrm{P}, \mathrm{T}, \mathrm{D}, \mathrm{A}, \mathrm{N}, \mathrm{C}, \mathrm{G}, \mathrm{E}, \beta, \mathrm{FM}, \mathrm{F}, \mathrm{S}, \mathrm{I}\right)$. Table 2 provides a summary of the FCPN-std definition. In FCPN-std, stochastic time information is incorporated in transitions and fuzzy information can be described in both - place and transition. Another advantage of FCPN-std is that it supports multiple color-token concept and modular design concept, which is discussed in Lee and Banerjee 2009. 


\section{Lee, Park and Banerjee}

Table 2: Definition of FCPN-std (Lee and Banerjee 2009)

\begin{tabular}{|c|c|c|}
\hline Notation & Meaning & $\begin{array}{ll}\text { Relations } \\
\end{array}$ \\
\hline$\sum$ & Set of color tokens & $\sum=\left\{\sigma_{1}, \sigma_{2}, \cdots\right\}$, where, $\sigma_{\mathrm{i}}=\mathrm{i}^{\text {th }}$ colored token \\
\hline$\Lambda$ & Set of distribution & $\Lambda=\left\{\right.$ Dist $_{1}$, Dist $\left._{2}, \cdots\right\}$ \\
\hline$P$ & Set of places & $\begin{array}{l}\mathrm{P}=\mathrm{P}_{\mathrm{g}} U \mathrm{P}_{\mathrm{f}} \text {, where } \mathrm{P}_{\mathrm{g}} \text { is the set of normal places and } \mathrm{P}_{\mathrm{f}} \text { is the set } \\
\text { of fuzzy places. }\end{array}$ \\
\hline $\mathrm{T}$ & Set of transitions & $\begin{array}{l}T=T_{g} U T_{f} \text {, where } T_{g} \text { is the set of normal transitions and } T_{f} \text { is the } \\
\text { set of fuzzy transitions. Normal transition can have stochastic } \\
\text { time delay and fuzzy transition can have fuzzy membership val- } \\
\text { ues. }\end{array}$ \\
\hline $\mathrm{D}$ & $\begin{array}{l}\text { Set of fuzzy description / } \\
\text { propositions }\end{array}$ & $\mathrm{D}=\left\{\operatorname{Des}_{1}, \operatorname{Des}_{2}, \cdots\right\}$ \\
\hline A & Set of arcs & $\mathrm{P} \cap \mathrm{T}=\mathrm{P} \cap \mathrm{A}=\mathrm{A} \cap \mathrm{T}=\varnothing$ \\
\hline $\mathrm{N}$ & Node function & $\mathrm{A} \rightarrow \mathrm{P} \times \mathrm{T} \mathrm{U} \mathrm{T} \times \mathrm{P}$ \\
\hline $\mathrm{C}$ & Color function & $\mathrm{PUT} \rightarrow \sum$ \\
\hline G & Guard function & $\forall \mathrm{t} \in \mathrm{T}:[\operatorname{Type}(\mathrm{G}(\mathrm{t}))=$ Boolean $\wedge \operatorname{Type}(\operatorname{Var}(\mathrm{G}(\mathrm{t}))) \subseteq \Sigma]$ \\
\hline $\mathrm{E}$ & Arc expression function & $\begin{array}{l}\forall \mathrm{a} \in \mathrm{A}:[\operatorname{Type}(\mathrm{E}(\mathrm{a}))=(\mathrm{C}(\mathrm{p}), \mathrm{R}) \wedge \operatorname{Type}(\operatorname{Var}(\mathrm{E}(\mathrm{a}))) \subseteq \\
\left.\sum\right], \text { where } \mathrm{R} \text { is real number to represent a capacity of arc. }\end{array}$ \\
\hline$\beta$ & Bijective function & $\mathrm{P}_{\mathrm{f}} \mathrm{U} \mathrm{T}_{\mathrm{f}} \rightarrow \mathrm{D}$ \\
\hline FM & $\begin{array}{l}\text { Set of Fuzzy membership } \\
\text { functions }\end{array}$ & $\mathrm{FM}=\left\{\mathrm{m}_{1}, \mathrm{~m}_{2}, \cdots\right\}$ \\
\hline $\mathrm{F}$ & Fuzzy mapping function & $\mathrm{D} \rightarrow \mathrm{FM}$ \\
\hline $\mathrm{S}$ & Stochastic mapping function & $\forall \mathrm{t} \in \mathrm{T}_{\mathrm{g}}:[\operatorname{Type}(\mathrm{S}(\mathrm{t}))=\Lambda \wedge \operatorname{Type}(\operatorname{Var}(\mathrm{S}(\mathrm{t}))) \subseteq \Sigma]$ \\
\hline I & Initial Marking & $\forall \mathrm{p} \in \mathrm{P}:[\operatorname{Type}(\delta(\mathrm{p}))=\mathrm{C}(\mathrm{p})]$ Where, $\delta: \mathrm{P} \rightarrow \sum$ \\
\hline
\end{tabular}

In this paper, the focus is on simulation methods of FCPN-std and described with an example. It is shown how the simulation and analysis of FCPN-std model can lead to the improvement of the modeled process..

\section{PROBLEM DESCRIPTION}

As an example of manufacturing process with uncertainties, two parallel processes with batch processors are considered. Figure 1 describes the example process to be modeled. This example consists of two parallel processes. Each parallel process is composed of three sub processes; (A, B, C) and (D, B', E). Sub processes B and B' are batch processes with different batch sizes. This type of setup is commonly encountered in semiconductor manufacturing processes such as wafer producing operations. In a semiconductor manufacturing process, sub processes A and D can be considered as upstream processes, and C and E can be considered downstream processes. Each parallel process handles two different product types $(\sigma 1$ and $\sigma 2)$.

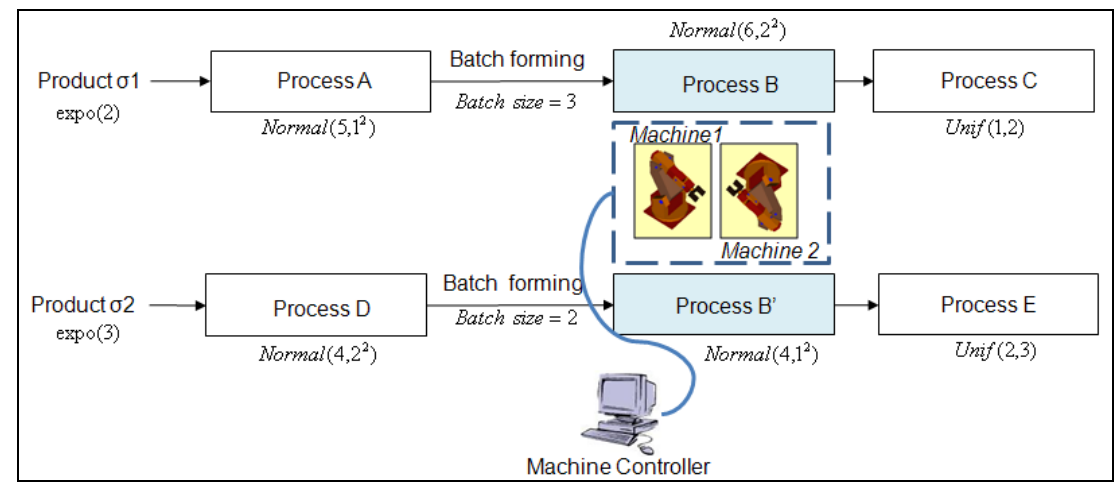

Figure 1: Two parallel processes with batch processors (time unit is in minutes )

Batch processes B and B' are handled by two identical machines; machine 1 and 2. Currently, machine 1 is used for process B only, and machine 2 is used for process B' only. In first process line, product type $\sigma 1$ arrives exponentially with interarrival time rate of 2 minutes. The bottleneck of first process line occurs in batch process B. Batch process B follows minimum batch size (MBS) policy. Similarly, the bottleneck in second line occurs in batch process B'. Also, process B' follows MBS rule. Based on two types of product's arrival distribution and processing time, batch process B encounters more fre- 


\section{Lee, Park and Banerjee}

quent bottlenecks subsequently machine 1 has less idle time. Using Monte Carlo simulation, performances of the two parallel processes are shown in table 3.

Table 3: System Performance of Current Manufacturing Processes (replication length $=1$ day; (8hours), replication $=30$ )

\begin{tabular}{|c|c|c|c|c|c|}
\hline & \multirow{2}{*}{$\begin{array}{c}\text { Cycle Time } \\
\text { (Minutes) }\end{array}$} & \multirow{2}{*}{ WIP } & \multicolumn{3}{|c|}{ Bottleneck in batch processes (Queue Size) } \\
\cline { 4 - 6 } & 21.95 & 5.9021 & Max & Average & Min \\
\hline Line1 & 2.9 & 2.43 & 0 \\
\hline Line2 & 10.61 & 2.7042 & 4 & 0.79 & 0 \\
\hline
\end{tabular}

From this simulation, designers can identify bottlenecks in both batch processes. Figure 2 shows the average queue length trends of in the batch processes indicating the bottlenecks during the simulation run.

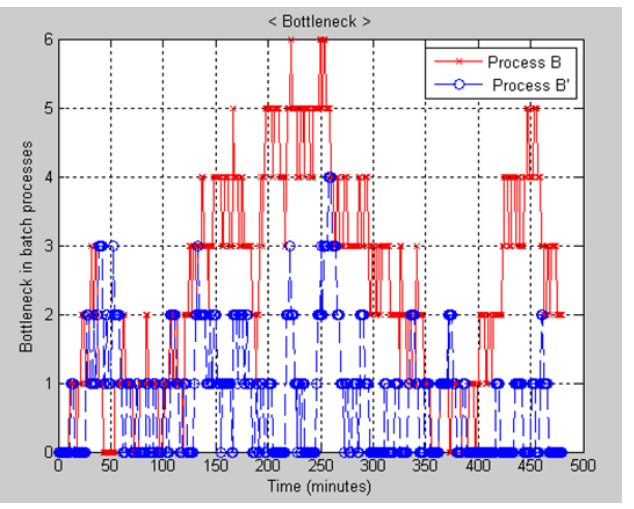

Figure 2: Bottleneck (Queue length) in two batch processes.

This bottleneck trend shows that batch process B has higher periods of bottleneck than process B', and machine 2 has more idle time. In order to improve these processes, process designers and engineers want to change control rules of machine 1 and 2. For example, one of the rules that is specified is in the following form: "if machine 2 is idle and machine 1 is busy, machine 2 can be used for process $B$ as well as process B' '. A machine's idleness can be measured using queue size of batch process. Table 4 describes 9 operation rules which are defined by process designers and engineers. For example, rule \#3 describes "If bottleneck status in process $B$ is high and bottleneck status in process B' is low then machine 2 can be used for both batch processes". It is perceived by the designers that bottlenecks could be decreased using these operation rules.

Table 4 : Operation Rules of Machine Controller for Improving System Performance

\begin{tabular}{|c|c|c|c|c|}
\hline \multirow{2}{*}{\multicolumn{2}{|c|}{ 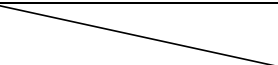 }} & \multicolumn{3}{|c|}{ Bottleneck Status in Process B } \\
\hline & & Low & Medium & High \\
\hline \multirow{3}{*}{$\begin{array}{l}\text { Bottleneck } \\
\text { Status } \\
\text { in } \\
\text { Process B' }\end{array}$} & Low & $\begin{array}{l}<\text { Rule \#1> } \\
\text { Process B : Machine1 } \\
\text { Process B' : Machine2 }\end{array}$ & $\begin{array}{l}<\text { Rule \#2> } \\
\text { Process B : Machine1, Machine2 } \\
\text { Process B': Machine2 }\end{array}$ & $\begin{array}{l}<\text { Rule \#3> } \\
\text { Process B : Machine1, Machine2 } \\
\text { Process B': Machine2 }\end{array}$ \\
\hline & Medium & $\begin{array}{l}<\text { Rule \#4> } \\
\text { Process B : Machine1 } \\
\text { Process B': Machine2, Machine1 }\end{array}$ & $\begin{array}{l}<\text { Rule \#5> } \\
\text { Process B : Machine1 } \\
\text { Process B': Machine2 }\end{array}$ & $\begin{array}{l}<\text { Rule \#6> } \\
\text { Process B : Machine1, Machine2 } \\
\text { Process B': Machine2 }\end{array}$ \\
\hline & High & $\begin{array}{l}<\text { Rule \#7> } \\
\text { Process B : Machine1 } \\
\text { Process B': Machine2, Machine1 }\end{array}$ & $\begin{array}{l}\text { Rule \#8> } \\
\text { Process B : Machine1 } \\
\text { Process B': Machine2 }\end{array}$ & $\begin{array}{l}<\text { Rule \#9> } \\
\text { Process B : Machine1 } \\
\text { Process B' : Machine2 }\end{array}$ \\
\hline
\end{tabular}

Since the targeted processes have fuzzy operation rules, stochastic processing time and multiple product types, it is difficult to model and analyze using existing methodologies. In the following sections, this manufacturing process is represented and simulated using the FCPN-std model. 


\subsection{Incorporating Manufacturing Uncertainties}

The example process has two types of uncertainties in processing time and machine control rules. Processing time in each sub process is captured using probability distribution. This distribution is acquired through past data history and statistical data fitting procedures. The distributions used in this example are shown in figure 1. Machine control rules are captured using fuzzy logic. As described in section 3, a machine's idleness is identified through bottleneck status. In the example, the bottleneck status is classified into 3 classes: low, medium, and high. By analyzing the current manufacturing process (as shown in table 3 and figure 2), the fuzzy membership functions are derived for bottleneck status of process B and B'. Bottleneck status of process B can be captured using these fuzzy sets:

- $\quad \operatorname{Low}=\{1 / 0,0.7 / 1,0.3 / 2,0 / 3\}$

- Medium $=\{0 / 1,1 / 2,0.8 / 3,0 / 4\}$

- $\operatorname{High}=\{0 / 2,0.3 / 3,0.8 / 4,1 / 5\}$

The expression of fuzzy set follows the representation by enumerating membership values and their elements in the set. For example, the first element of the Low set (1/0) represents that the element 0 has the fuzzy membership value 0 . This representation can be replaced by the mathematical function form using indicator function. The bottleneck status of process B' are described using the fuzzy sets mentioned below:

- $\quad$ Low $=\{1 / 0,0.3 / 1,0 / 2\}$

- $\quad$ Medium $=\{0 / 0,1 / 1,0.6 / 2,0 / 3\}$

- $\operatorname{High}=\{0 / 1,0.3 / 2,0.8 / 3,1 / 4\}$

Figure 3 shows the fuzzy membership function plots with respect to the bottleneck of batch processes. In figure 3 (a), the $\mathrm{X}$ axis represents the waiting queue size of process $\mathrm{B}$ and $\mathrm{Y}$ axis describes the fuzzy utility values with respect to the queue size. For instance, if the waiting queue size in process $\mathrm{B}$ is 2 , the low, medium, and high have $0.3,1$, and 0 values respectively. Using fuzzy inference rule, medium status is selected since it has the maximum fuzzy utility value.

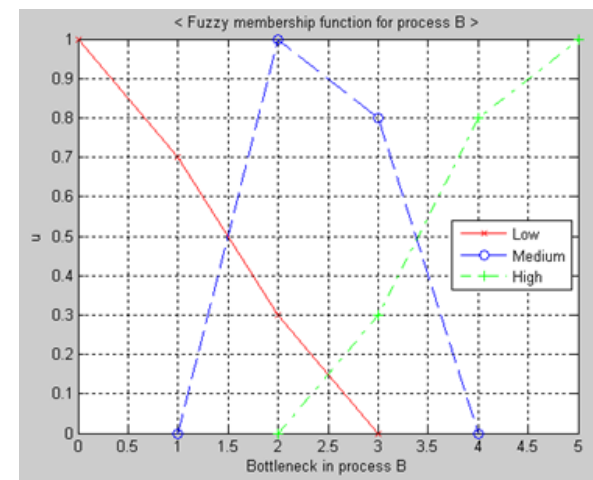

(a)Fuzzy membership functions for process B

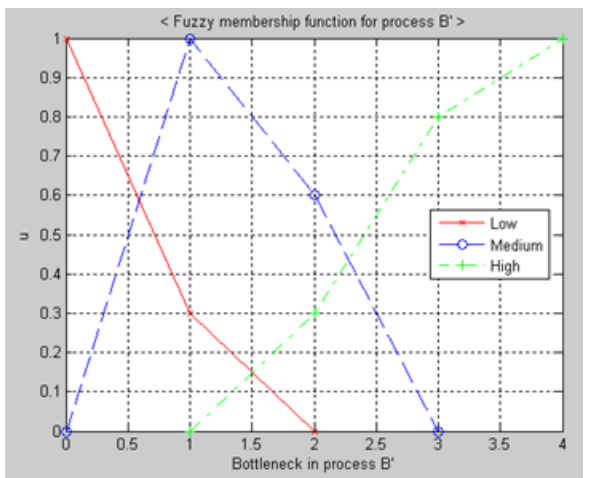

(b) Fuzzy membership functions for process B'

Figure 3: Plots of fuzzy membership functions.

These fuzzy sets and characteristics of fuzzy membership functions can be varied with respect to characteristics of manufacturing process and improvement targets/objectives. As the fuzzy membership functions are driven from if-clause, another fuzzy membership function is needed to describe then-clause. In rule \#3 (table 4), machine 2 can be used by process B or process B'. This situation is also captured by a fuzzy membership function as illustrated in figure 4. It describes machine 2's control strategy in the case when the bottleneck of process B is high and the bottleneck of process B' is low. In this function, $\mathrm{X}$ axis has real number with interval $[0,1]$. 0 signifies that machine 2 is being used for process $\mathrm{B}$, and 1 signifies that machine 2 is being used for process B'. Using the suggested fuzzy inference rule as described in section 5.1, the defuzzificated value is rounded and process which machine will be used for can be selected. These fuzzy membership function is characterized by manufacturing operators or process engineers. 


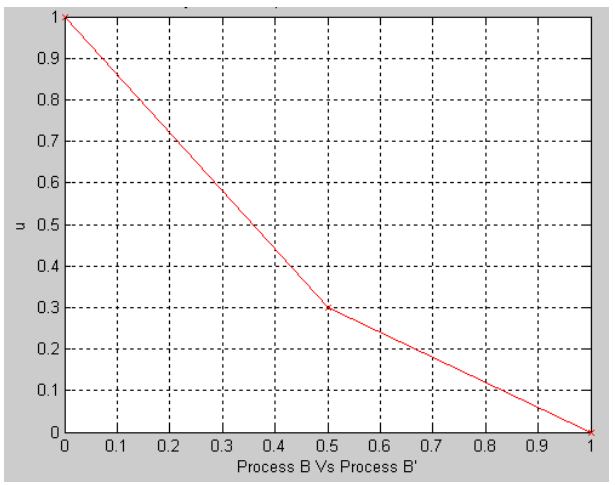

Figure 4: Fuzzy membership function for machine 2's working process

\subsection{Representation of FCPN-std Model}

The uncertainties described in section 3 are incorporated into FCPN-std using fuzzy logic and probability distribution . As mentioned in section 2, a FCPN's place and transition can have fuzzy description/proposition $\left(\mathrm{D}=\left\{\operatorname{Des}_{1}, \operatorname{Des}_{2}, \cdots\right\}\right)$ using bijective function $\beta\left(\beta: \mathrm{P}_{\mathrm{f}} \mathrm{U} \mathrm{T}_{\mathrm{f}} \rightarrow \mathrm{D}\right)$. This proposition can be used as a representation of if-else-then clauses for fuzzy rules. This proposition may consist of sub propositions as well. Each sub proposition (for example, Des ${ }_{5}$ ) has fuzzy membership function (m1; $\left.F M=\left\{\mathrm{m}_{1}, \mathrm{~m}_{2}, \cdots\right\}\right)$ using fuzzy mapping function $\mathrm{F}(\mathrm{F}: \mathrm{D} \rightarrow \mathrm{FM})$. A transition can have stochastic time delay. These information are represented with the color token concept. In this example, four types of color tokens are used. A circle-type token represents product 1 and a plus-type token represents product 2 . A triangle-type token depicts machine 1 and a box-type token describes machine 2. Based on these tokens, places can have multiple interpretations. Figure 5 shows the FCPN-std model of this example. Place $\mathrm{p} 1$ represents start position of process A or D depending on token types. If token type is circle, place 1 is considered as a starting status of process A. Otherwise, it is considered as a starting status of process D. The detailed definition and representation follows the definition of FCPN-std in (Lee and Banerjee 2009). The following section describes how this FCPN-std model is simulated and analyzed.

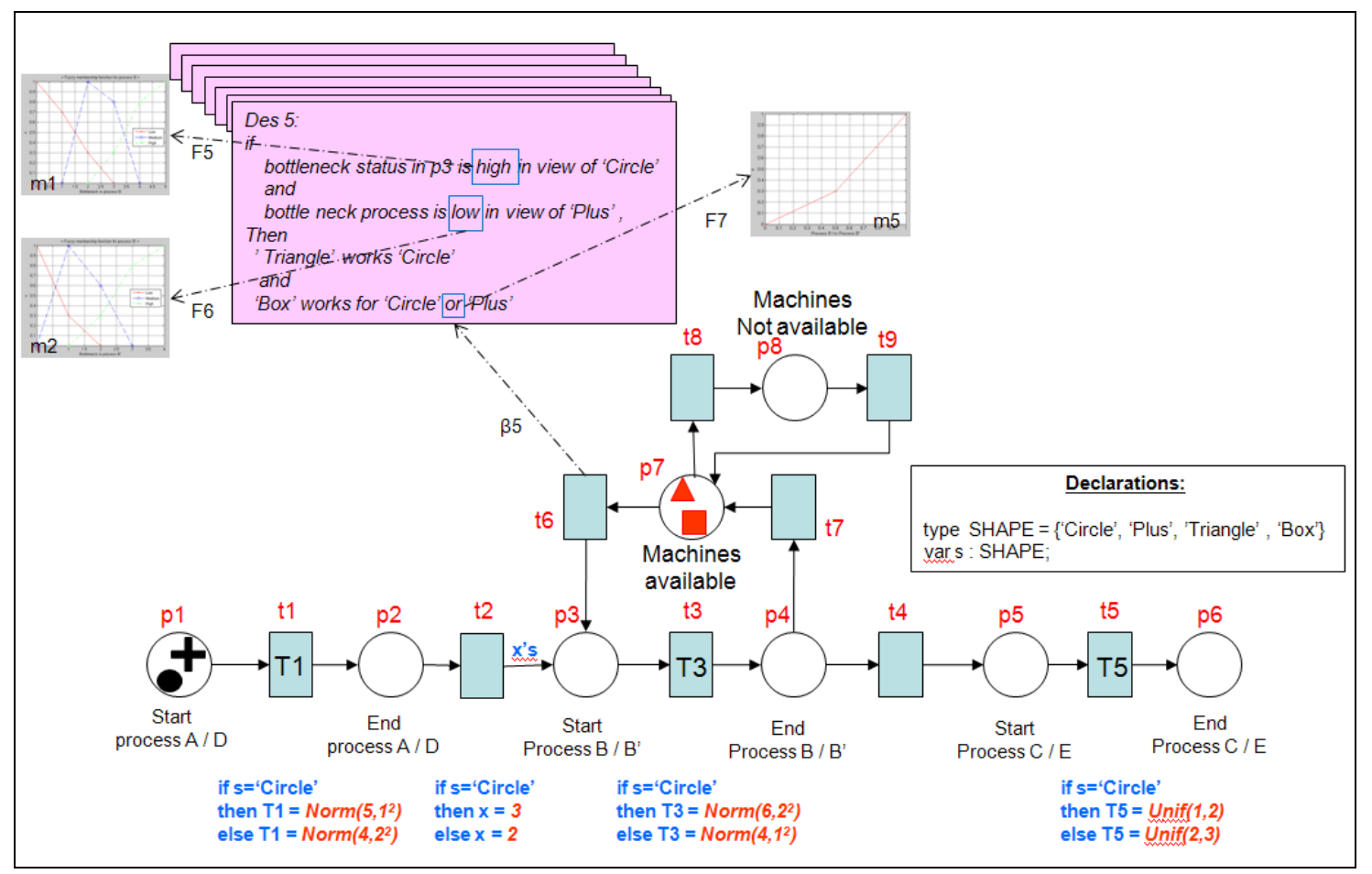

Figure 5: FCPN-std model of processes used in the example 


\section{EXECUTION AND SIMULATION OF FCPN-STD MODEL}

This section describes the simulation methodology of FCPN-std model. In this section, new inference rule and defuzzification method are described for executing an effective simulation. This is followed by the simulation procedure and results.

\subsection{Fuzzy Inference Rule and Defuzzification Method}

General fuzzy inference is executed through 4 stages: fuzzy matching, inference, combination, and defuzzification (Yen and Langari 1999). In the fuzzy matching stage, each fuzzy degree in if-clause is calculated. Based on these fuzzy degree, the ifclause's conclusion is generated as a format of fuzzy membership function. It is used for drawing the fuzzy degree of thenclause. Finally, the decision is made using defuzzification methods such as Mean of Maximum (MOM) method or Center of Area (COA) methods. Figure 6 shows the general procedure of fuzzy inference execution. This execution method varies based on a model's characteristics and problem domain.

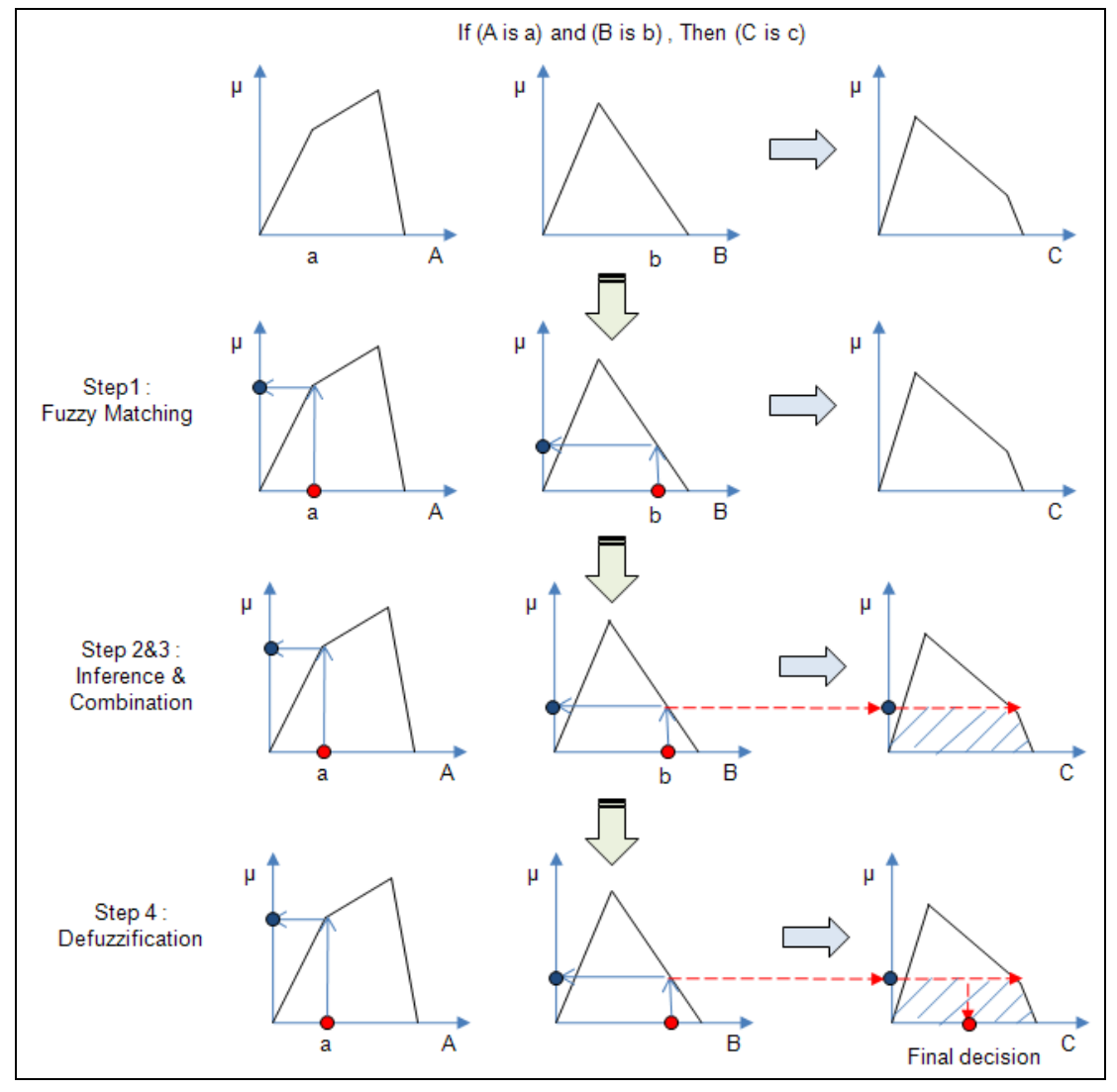

Figure 6: General procedures of fuzzy inference execution

In this generated FCPN-std model, fuzzy inference rules are executed differently. As described in table 4, each fuzzy inference rule has the same format: "If (A) is (a) and (B) is (b), then (C) is (c) and (D) is (d)".

For example, fuzzy inference rule 3 says that "if the bottleneck status of process B (A) is high (a) and bottleneck status of process $B^{\prime}$ (B) is low (b), then machine 1 (C) is used for process $B$ (c) and machine 2 (D) can be used for both process $B$ and $\underline{B^{\prime}}(\mathbf{d}) "$.

In this format of fuzzy inference rule, the clause (A is a) is called as a "action condition" and (B is b) is called as a "guiding condition". ( $\mathrm{C}$ is c) and (D is d) is classified into "normal action" and "fuzzy action". By checking the bottleneck status of both process B and B', the fuzzy degree of action condition and guiding condition is determined. Based on the two fuzzy degree values, the current fuzzy inference rule is determined. When the fuzzy inference rule is selected, only guiding condition and fuzzy action are considered. The fuzzy degree of guiding condition is used for calculating fuzzy degree of fuzzy action. The calculated fuzzy degree is defuzzificated using MOM method. Finally, the machine's working process is determined using rounding defuzzificated value. Table 5 describes classified conditions and actions per each fuzzy inference rule. 


\section{Lee, Park and Banerjee}

Table 5: Fuzzy Inference Rule and its Classification

\begin{tabular}{|c|c|c|c|c|}
\hline \multirow{2}{*}{$\begin{array}{c}\text { Fuzzy } \\
\text { Inference } \\
\text { Rule }\end{array}$} & Bottleneck status in B & Bottleneck status in B' & $\begin{array}{c}\text { Then statement } \\
\text { Machine 1 } \\
\text { Works for process }\end{array}$ & $\begin{array}{c}\text { Machine 2 } \\
\text { works for process }\end{array}$ \\
\hline 1 & Low (G) & Low $(\mathrm{G})$ & B (N) & B'(N) \\
\hline 2 & Medium (G) & Low (A) & B (N) & B \& B'(F) \\
\hline 3 & High (G) & Low (A) & B (N) & B \& B'(F) \\
\hline 4 & Low (A) & Medium (G) & B \& B'(F) & B (N) \\
\hline 5 & Medium (G) & Medium (G) & B (N) & B'(N) \\
\hline 6 & High (G) & Medium (A) $(\mathrm{N})$ & B \& B'(F) \\
\hline 7 & Low (A) & High (G) & B \& B'(F) & B (N) \\
\hline 8 & Medium (G) & High (G) & B (N) & B'(N) \\
\hline 9 & High (G) & High (G) & B (N) & B'(N) \\
\hline
\end{tabular}

\{G: Guiding condition, A: Action condition, N: Normal action, F: Fuzzy action\}

Figure 7 shows the overall procedure of fuzzy inference execution in this FCPN-std model. When place p3 (in figure 5) has only 4 Circle-shape tokens, the $3^{\text {rd }}$ fuzzy inference rule is selected by evaluating fuzzy membership function. Using table 5 , the action condition and fuzzy action are extracted. From MOM defuzzification and rounding, the future action (Machine 2 to be used for process B for reducing bottleneck in B) is fulfilled. Using this process, the high bottleneck status of process B can be potentially decreased.

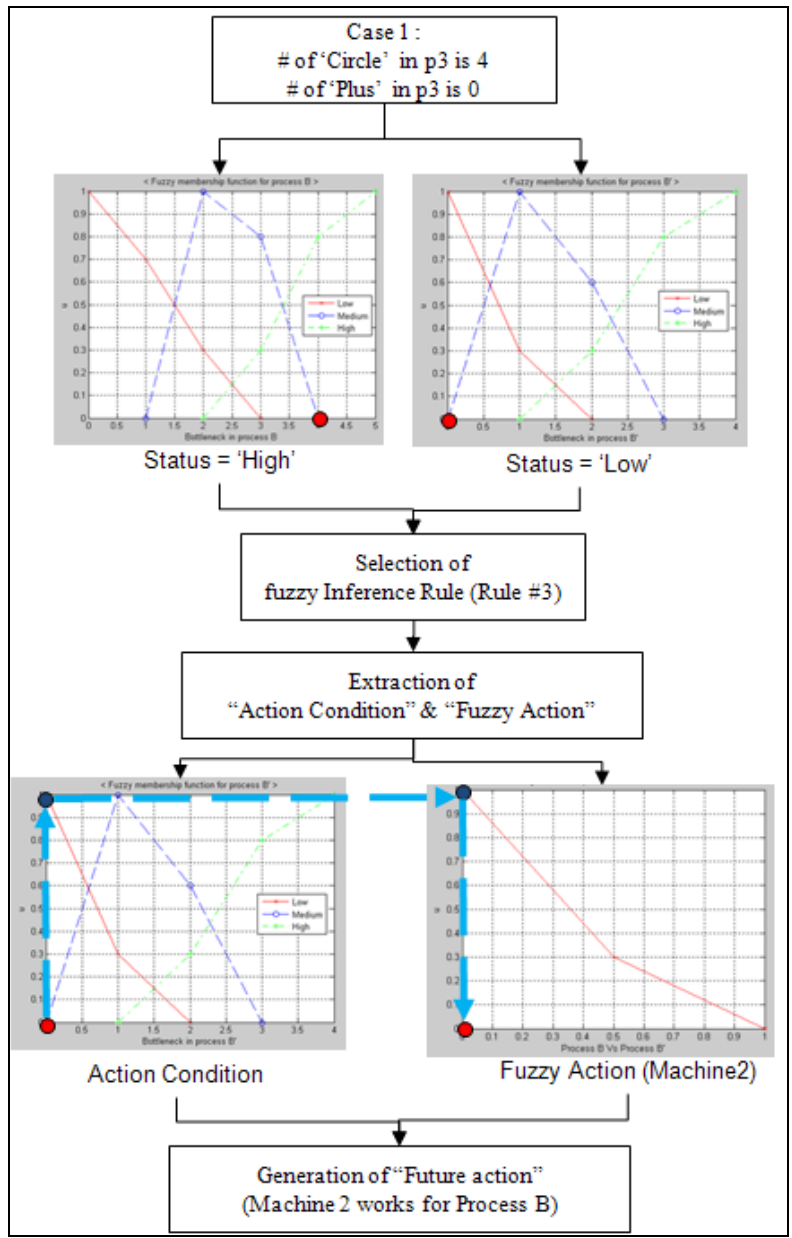

Figure 7 : Execution of fuzzy inference rule 


\section{Lee, Park and Banerjee}

\subsection{Simulation and Results of FCPN-std model}

Using the method described in section 5.1, the batch processor generates and controls each machine's action. Through generated future actions, transitions are enabled and fired and matched tokens are moved. If a transition has stochastic time delay, the token is delayed at the transition using a random variate generated from the underlying probability distribution. The remaining simulation follows the general simulation rule of CPN (Jensen, Kristensen and Wells 2007) and Monte Carlo simulation procedure. Table 6 shows the simulation results of this FCPN-std model.

Table 6: System Performance of FCPN-std model (replication length $=1$ day; $(8$ hours $)$, replication $=30$ )

\begin{tabular}{|c|c|c|c|c|c|}
\hline & \multirow{2}{*}{$\begin{array}{c}\text { Cycle Time } \\
\text { (Minutes) }\end{array}$} & \multirow{2}{*}{ WIP } & \multicolumn{3}{|c|}{ Bottleneck in batch processes (Queue Size) } \\
\cline { 4 - 6 } & & & Max & Average & Min \\
\hline \multirow{2}{*}{ Line1 } & 21.87 & 5.89 & 6 & 2.41 & 0 \\
& $(21.95)$ & $(5.90)$ & $(6)$ & $(2.43)$ & $(0)$ \\
\hline \multirow{2}{*}{ Line2 } & 10.22 & 2.62 & 4 & 0.72 & 0 \\
& $(10.61)$ & $(2.70)$ & $(4)$ & $(0.79)$ & $(0)$ \\
\hline
\end{tabular}

Values in () represents the system performance before applying FCPN-std.

This result shows that the system performance has slightly improved after applying fuzzy operation rule. The system performance can be further improved through better control of used fuzzy membership functions.

\section{CONCLUSION AND DISCUSSION}

This paper proposed a new and efficient method for modeling, simulating and analyzing manufacturing uncertainties. Manufacturing uncertainties are classified into information with variance and information with vagueness. Information with variance are captured using probability distribution and information with vagueness are captured using fuzzy logic. These uncertainties are incorporated into FCPN-std. FCPN-std model can handle general uncertainties and supports colored token concept. With these characteristics, the targeted manufacturing process can be modeled without creating distortions in the form of approximation and ignoring of uncertainties. It helps designers interpret their targeted process clearly and precisely.

The simulation of FCPN-std model is fulfilled by the execution of incorporated fuzzy inference rule and delay of stochastic time. The execution of incorporated fuzzy inference rule is started from the evaluation of fuzzy membership functions. Their executions vary based on process domain knowledge and design objectives. After acquiring actions from execution algorithms, the FCPN-std model is simulated using Monte Carlo simulation methodology.

As further studies, control of fuzzy membership functions needs to be considered. The system performance of FCPN-std is influenced by manufacturing process itself, the stochastic time delay characteristics and incorporated fuzzy inference rule. Among them, the fuzzy inference rule can be an important key for improving processes. As more manufacturing processes are becoming complex, it is getting difficult to apply mathematical programming to obtain optimal solution or queuing theory. In such cases, a FCPN-std model can be an alternative test bed for process improvement. Also, the file format and tool for FCPN-std needs to be developed and standardized for convenient usage and data exchange.

\section{REFERENCES}

Lee, H. and Banerjee, A. 2009. Modeling and Representation of Manufacturing Process with Uncertainties Using Parametric Modular Design and xPNML. Proceedings of the 2009 International Symposium on Collaborative Technologies and Systems, Baltimore, MD, 512-519.

Kumar, R., Kumar, S. and Tiwari, M.K. 2005. An expert enhanced coloured fuzzy Petri net approach to reconfigurable manufacturing systems involving information delays. International Journal of Advanced Manufacturing Technology . Vol 26, 922-933.

Bause, F. and Kritzinger, P.S. 2002. Stochastic Petri Nets : An Introduction to the theory. Vieweg.

Raposo, A., Coelho, A., Magalhaes, L. and Ricarte, I. 2001. Using Fuzzy Petri Nets to Coordinate Collaborative Activities. Proceedings of the Joint 9th IFSA World Congress and 20th NAFIPS International Conference, Vancouver, Canada, 1494-1499.

Buzacott, J.A. and Shanthikumar, J.G. 1993. Stochastic Models of Manufacturing Systems. Prentice Hall.

Hopp, W.J. and Spearman, M.L. 1993. Factory Physics : Foundations of Manufacturing Management. Irwin-McGraw Hill. 


\section{Lee, Park and Banerjee}

Yen, J. and Langari, R. 1998. Fuzzy Logic: Intelligence, Control, and Information. Prentice-Hall.

Zhou, M. and Venkatesh, K. 1999. Modeling, Simulation, and Control of Flexible Manufacturing Systems. World Scientific.

Yeung, D.S., Liu, J., Shiu, S. and Fung, G. 1996. Fuzzy Coloured Petri Nets In Modeling Flexible Manufacturing Systems. ISAI/IFIS '96, 100-107.

Cardoso, J. and Camargo, H. 1999. Fuzziness in Petri Nets. In Studies in Fuzziness and Soft Computing Physica-Verlag.

Jensen, K., Kristensen, L.M. and Wells, L. 2007. Coloured Petri Nets and CPN Tools for modelling and validation of concurrent system. Int $J$ Software Technology Transfer 9, 213-254.

\section{AUTHOR BIOGRAPHIES}

HYUNSOO LEE is a Doctoral student in the Department of Industrial and Systems Engineering at Texas A\&M University. He received his master's degree from Pohang University of Science and Technology (POSTECH), Korea, and worked as a $\mathrm{CAD} / \mathrm{PLM} / \mathrm{CPC}$ consultant in SAMSUNG for 5 years. His research interests are in intelligent manufacturing simulation, cooperative design and manufacturing, virtual model ontology and pattern classification and tracking. His email is $<$ hsleneo.tamu.edu>.

HONGSUK PARK is a Doctoral student in the department of Industrial and Systems Engineering. He completed his Master's degree from TEPP(Techno-Economics Policy Program) in Seoul National University and his B.S. degree from Weapons Engineering at Korea Military Academy. He worked as a military officer and helicopter pilot for 11 years. Since 2005, he worked as a Defense Technology Management Officer at Project Management Groups, ROKA Headquarters. His research interest lies in the Management of Defense Technology including Modeling and Simulation. His email is <avipaketamu.edu>.

AMARNATH BANERJEE is an Associate Professor and the Director of Undergraduate Program in the Department of Industrial and Systems Engineering at Texas A\&M University. He received his Ph.D. in Industrial Engineering and Operations Research from the University of Illinois at Chicago, and a BS in Computer Science from Birla Institute of Technology and Science in India. His research interests are in virtual prototyping and manufacturing, simulation, image processing, real-time video processing, augmented reality and human behavior modeling. He directs the Virtual and Augmented Reality Laboratory. He teaches courses in manufacturing and production systems design and control, facilities planning, intelligent systems, virtual manufacturing and simulation. He is an Associate Editor of International Journal of Services, Operations and Informatics. He serves on the Advisory Council of the Rural and Community Health Institute, a part of Texas A\&M Health Science Center. He is a member of IEEE and a senior member of the Institute of Industrial Engineers (IIE). His email address is $<$ banerjeedtamu.edu $>$. 